\title{
Colon cleansing protocol in children: research conditions vs. clinical practice
}

\section{(ㄷ)(1) $\odot$}

\author{
Authors \\ Yoram Elitsur, Yaslam Balfaqih, Deborah Preston \\ Institution \\ Joan C Edwards School of Medicine at Marshall University - \\ Pediatrics, Huntington, West Virginia, United States \\ submitted 26.5.2017 \\ accepted after revision 20.10.2017 \\ Bibliography \\ DOI https://doi.org/10.1055/s-0043-121985 | \\ Endoscopy International Open 2018; 06: E410-E413 \\ (c) Georg Thieme Verlag KG Stuttgart · New York \\ ISSN 2364-3722 \\ Corresponding author \\ Yoram Elitsur, MD, Department of Pediatrics, 1600 Medical \\ Center Drive, Huntington, WV 25701 \\ Fax: +1-304-691-1375 \\ elitsur@marshall.edu
}

\section{ABSTRACT}

Background and study aims Colon preparation rates are the limiting factor for a successful diagnostic colonoscopy in children. Different colon cleansing protocols have been published for use in children. Unfortunately, the applicability of those published research protocols has not been for- mally evaluated in routine clinical practice. We investigated the success rate of our previously published colon cleansing protocol as utilized in our clinical practice.

Patients and methods This was a retrospective study. In the clinical practice, the colon cleansing protocol included PEG-3350 at a dose of $2 \mathrm{~g} / \mathrm{kg} /$ day plus Dulcolax (Bisacodyl, Boehringer Ingelheim, TX USA) $5 \mathrm{mg} /$ day for 2 days. Adequate colon preparation was graded between $1-5$, as previously described, and grade $\geq 4.0$ was considered an adequate preparation. Patients were instructed to complete a questionnaire that included PEG-3350 dose, number of stools per day, consistency of each stool, and side effects (vomiting, abdominal pain). Clinical and endoscopic results were compared between the protocol under research conditions and routine practice.

Results The success rate of the colon preparation in our clinical practice was similar to the results observed under our research protocol (75\% vs. $73.6 \%$ ). Moreover, the total number of stools, stool consistency, and the intubation rate of the terminal ileum were also similar. We concluded, that in our experience, the colon cleansing protocol used under research conditions was effective and appropriate for use in routine clinical practice.

Conclusion We recommend testing each new protocol under the routine conditions of clinical practice to confirm its applicability for general practitioners.

\section{Introduction}

Colonoscopy in children has become a crucial diagnostic tool for various colonic diseases such as rectal bleeding, chronic diarrhea, inflammatory bowel disease, and more. Satisfactory colon preparation is considered a major limiting factor in achieving adequate colon conditions to assess mucosal appearance, histology, and/or to perform different surgical procedures. Colon cleansing protocols adopted from adult practices were not appropriate for children for various reasons, including undesirable flavor and the large volume needed [1]. In recent years, different pediatric PEG-3350-based colon cleansing protocols were reported in the literature, which differed in length of preparation (1 to 4 days) and laxative dose $(1-4 \mathrm{~g} / \mathrm{Kg} / \mathrm{d}$ ) [2 $6]$. In accordance with research guidelines, in all cited prospec- tive studies, protocols were performed under strict research conditions. To our knowledge, the corresponding authors of those protocols have not provided evidence to confirm that their published protocols are successful under normal clinical conditions. Accordingly, the applicability of those protocols for general gastroenterologists is unproven. We believe that a successful research protocol should be used routinely by the authors in their normal clinical practices to show the suitability of their research protocol for clinical practice. This supposition is supported by overall failure rates for different cleansing protocols in children, which were estimated in different studies between $21 \%$ and $34 \%$ [7-11]. Whether the high rate of unprepared colons reflects the failure of a previously published research protocol or new protocol used by the respected authors is not known. It is obvious that assessing research protocols in 
- Table 1 Colon cleansing results.

\begin{tabular}{|l|l|l|l|}
\hline & Clinical practice & Research protocol [11] & P value \\
\hline Number of patients & 89 & 38 & $9.9 \pm 4.7$ \\
\hline Age (years) (mean + SD) & $13.9 \pm 4.2$ & $0.8: 1.0$ & 0.6648 \\
\hline M/F ratio & $1.5: 1.0$ & $7.5 \pm 4.2$ & 0.920 \\
\hline Number of stools/day (mean+SD) & $7.9 \pm 4.1$ & $5.6 \pm 0.63$ & 0.6292 \\
\hline Stool consistency grade (mean+SD) & $5.6 \pm 0.66$ & $4.1 \pm 0.79$ & 0.8275 \\
\hline Colon cleansing grade (mean + SD) & $4.0 \pm 0.94$ & $28(73.7 \%)$ & 0.4992 \\
\hline Colon cleansing grade $>4.0$ & $67(75.3 \%)$ & $36(94.7 \%)$ & \\
\hline Tl intubation & $72(88.9 \%)$ & & \\
\hline M, male; F, female; SD, standard deviation; TI, terminal ileum & & \\
\hline
\end{tabular}

routine clinical practices may reduce the rate of failed colon preparation in the practices of general gastroenterologists.

In 2013, we published a prospective research study that compared the success rate of colon preparations between 2and 4-day colon cleansing protocols in children. In that study, other colonoscopy landmarks were measured including: terminal ileum ( $\mathrm{TI}$ ) intubation rate and clinical side effects (vomiting, abdominal pain). Our results showed an adequate preparation rate of $73.6 \%$ after the 2 -day protocol [11].

Since that publication, we have incorporated this protocol into our routine clinical practice and collected data from a suitable number of procedures to be able to report our results. Accordingly, in the current study, we compared the colon preparation rate between the strict research protocol and the same protocol performed under routine clinical practice.

\section{Patients and methods}

In this retrospective study, the colon cleansing protocol included PEG-3350 at a dose of $2 \mathrm{~g} / \mathrm{kg} /$ day plus Dulcolax (Bisacodyl, Boehringer Ingelheim, Texas, United States) $5 \mathrm{mg} /$ day as previously described [11]. Patients were instructed to mix $8 \mathrm{oz}$ of liquid for each $17 \mathrm{~g}$ of PEG 3350 consumed, provided that the liquid was not red. Patients also completed a questionnaire that included the amount of PEG-3350 consumed per day, the number of stools per day, the consistency of each stool (graded previously as: G1-hard stool, G2-firm stool, G3-curd form stool, G4-soft stool, G5-loose/liquid stool, G6-watery/thin stool [3], and side effects (vomiting, abdominal pain). All patients who underwent colonoscopies for different medical reasons were included in the study. No exclusion criteria were used. Adequate colon preparation was defined according to Vanner S] [12] and Barclay et al. [13] with modifications (G1 unacceptable, G2-poor, G3-fair, G4-good, G5-excellent). Colon preparation of grade $\geq 4$ was considered adequate preparation in both studies (research and routine clinical practice). Implementation of the research protocol differed from that in routine clinical setting in the following ways: 1 . Patients were instructed to call in for any protocol deviations; 2 . Review of pa- tients' questionnaires was performed before the procedures; 3 . Colon preparation grading was also assessed independently by the endoscopic nurse; and 4. Final preparation grading was calculated as the average of grading of both reviewers: the gastroenterologist (YE) and the endoscopy nurse. The study was approved by the Marshall University School of Medicine Institutional Review Board.

\section{Results}

A total of 89 colon cleansing protocols were performed in children aged 3 to 22 years. The most common indications for colonoscopy were gastrointestinal bleeding or inflammatory bowel disease in patients undergoing colonoscopy as part of their clinical investigation or follow-up. The patients' average age was 13.9 years and the male to female ratio was 1.53:1.0 ( $\triangleright$ Table 1). As expected, abdominal cramps related to the PEG 3350 were noted in 41 patients (46\%), which did not result in any protocol violations. Vomiting was noted in 3 (3.3\%) patients. Similar results were noted in the previous study, showing abdominal cramps in $32 \%$ and vomiting $2.5 \%$ [11]. No patients failed to complete the colon cleansing protocol due to side effects. Overall, the adequate colon preparation rate $(\geq 4.0)$ was $75 \%$ and successful terminal ileum ( $\mathrm{TI}$ ) intubation was noted in $89 \%$ of the procedures. Incomplete TI intubation was noted in 10 procedures of which inadequate colon cleansing was noted in 3 patients, no $\mathrm{Tl}$ in 1 patient (post-surgery), anatomical stenosis in 5 patients, and failed intubation in $1 \mathrm{pa}$ tient. Under research conditions, incomplete TI intubation was noted in 2 children, both due to anatomical stenosis. The odds of achieving adequate colon cleansing were similar regardless of whether it was performed under research conditions or clinical practice (OR 1.1; Cl $95 \% 0.4-2.5 ; P=0.8275$ ). Chi-square and Fisher's Exact Test were used to compare Colon Cleansing Grading ( $\geq 4)$ and TI Intubation rate. One-way ANOVA and Mann-Whitney was used to compare the number of stools/day, stool consistency, and the average of the colon cleansing grading. The odds ratio, confidence interval, and $P$ values in the results were calculated using Chi-square and Fisher's Exact test 
using the Baptista-Pike method. All statistics were calculated using Graph Pad Prism, version 7.03, by GraphPad Software, Inc., LaJolla, Ca, USA.

\section{Discussion}

Colon preparation is the crucial limiting factor for successful evaluation of the colon, as well as for diagnostic purposes or surgical procedures. In a review of over 12,800 colonoscopies in adults, colon cleansing quality was a decisive factor in detection of polyps [14]. Others reported that repeated colonoscopies on previously inadequate colon preparations were successful in only $90 \%$ of cases, while $10 \%$ were inadequately prepared the second time [15]. Different reports in children showed that an inadequate preparation rate ranged between $21 \%$ and $34 \%$ [7-10]. The wide range of unprepared colons in children is probably related to the different cleansing protocols utilized in each study, and existed in spite of the close monitoring of the patients during the respective, prospective research protocols. Incomplete colonoscopies are also associated with a significant burden on the patients and their families due to the psychological effects, anesthesia involved, and extra medical expenses related to repeat procedures [16]. Another factor that has not been evaluated on this topic is the practicality of the suggested cleansing protocols used in research studies, namely, none of the respective authors have provided documentation that their protocols would be successful in real-life practices. This is an important point, as the real value of any research protocol is its practical use in the clinical practices of ordinary gastroenterologists, who perform their cleansing protocols without the strict conditions of research protocols. Without such proof, those cleansing protocols performed under research conditions could be lost in the literature and may never reach "real world practice."

We acknowledge that the limitation of our study was mainly that the number of patients in the two groups were not comparable in size. The research protocol only had 38 patients participate compared to the 89 patients from the clinical practice. Nonetheless, no significant differences were noted between the groups.

\section{Conclusion}

In summary, we presented in this study data from our previously published prospective 2 -day colon cleansing research protocol and compared the results to the same procedure used under the conditions of our routine clinical practice. In this retrospective study we showed that the colon preparation rate was similar to the result obtained under the previous research conditions and confirmed that this protocol is applicable for use in routine clinical practices. Other colonoscopy landmarks including intubation of the $\mathrm{TI}$ and side effects were also similar in both protocols. To our knowledge, this is the first comparison of the same colon cleansing protocol under research conditions and routine clinical practice reported in children. We recommend that physicians who suggest a new colon cleansing protocol provide follow-up data to show that their protocol can be uti- lized in their own routine clinical practice; i. e.: without the restrictions associated with research protocols. This information will demonstrate that their protocol is applicable for physicians in other non-academic medical centers.

Without such proof, the published research protocols could be buried in the medical literature and never be practiced in the real world.

\section{Competing interests}

None

References

[1] Safder S, Demintieva Y, Rewalt M et al. Stool consistency and stool frequency are excellent clinical markers for adequate colon preparation after polyethylene glycol 3350 cleansing protocol: a prospective clinical study in children. Gastrointest Endosc 2008; 68: 1131-1135

[2] Di Nardo G, Aloi M, Cucchiara S et al. Bowel preparations for colonoscopy: an RCT. Pediatrics 2014; 134: 249-256

[3] Abbas MI, Nylund CM, Bruch C] et al. Prospective evaluation of 1 day polyethylene glycol 3350 bowel preparation regimen in children. J Pediatr Gastroenterol Nutr 2013; 56: 220-224

[4] Pashankar DS, Uc A, Bishop WP. Polyethylene glycol 3350 without electrolytes: a new safe, effective, and palatable bowel preparation for colonoscopy in children. Pediatrics 2004; 144: $358-362$

[5] Phatak UP, Johnson S, Husain SZ et al. Two-day bowel preparation with polyethylene glycol 3350 and bisacodyl: a new, safe, and effective regimen for colonoscopy in children. J Pediatr Gastroenterol Nutr 2011; 53: $71-74$

[6] Pall H, Zacur GM, Kramer RE et al. Bowel preparation for pediatric colonoscopy: Report of the NASPGHAN endoscopy and procedures committee. J Pediatric Gastroenterol Nutr 2014; 59: 409-416

[7] Gremse DA, Sacks Al, Raines S. Comparison of oral sodium phosphate to polyethylene glycol-based solution for bowel preparation for colonoscopy in children. J Pediatr Gastroenterol Nutr 1996; 23: 586 - 590

[8] Barrish JO, Gilger MA. Colon cleanout preparations in children and adolescents. Gastroenterol Nurs 1993; 16: 106-109

[9] Dahshan A, Lin CH, Peters J et al. A randomized, prospective study to evaluate the efficacy and acceptance of three bowel preparations for colonoscopy in children. Am J Gastroenterol 1999; 94: 3497-3501

[10] da Silva MM, Briars GL, Patrick MK et al. Colonoscopy preparation in children: safety, efficacy, and tolerance of high- versus low-volume cleansing methods. J Pediatr Gastroenterol Nutr 1997; 24: 33-37

[11] Elitsur R, Butcher L, Lund V et al. Colon preparation in children: head to head comparison between 2 and 4 days PEG 3350 protocols. World J Gastrointestinal Endosc 2013; 5: 165-168

[12] Vanner SJ, MacDonald PH, Paterson WG et al. A randomized prospective trial comparing oral sodium phosphate with standard polyethylene glycol-based lavage solution (Golytely) in the preparation of patients for colonoscopy. Am J Gastroenterol 1990; 85: 422 - 427

[13] Barclay RL, Depew WT, Vanner S]. Carbohydrate-electrolyte rehydration protects against intravascular volume contraction during colonic cleansing with orally administered sodium phosphate. Gastrointest Endosc 2002; 56: 633-638

[14] Radaelli F, Meucci G, Sgroi G et al. Technical performance of colonoscopy: the key role of sedation/analgesia and other quality indicators. Am J Gastroenterol 2008; 103: $1122-1130$ 
[15] Ibáñez M, Parra-Blanco A, Zaballa P et al. Usefulness of an intensive bowel cleansing strategy for repeat colonoscopy after preparation failure. Dis Colon Rectum 2011; 54: $1578-1584$
[16] Kesteloot K, Veereman-Wauters G, Nolis I et al. A model for calculating the hospital costs of pediatric endoscopy. Gastrointest Endosc 1996; 44: $548-553$ 